\title{
Redundancy Optimization Based on Compressive Sensing for Industrial Wireless Sensor Networks
}

\author{
Ju Yun", Chen Quanhe, Mazin Yousif \\ School of Control and Computer Engineering, North China Electric Power University, 102206, China
}

\begin{abstract}
A routing algorithm, based on a dual cluster head redundant mechanism combined with compressive sensing data fusion algorithm, is proposed to improve reliability and reduce data redundancy of the industrial wireless sensor networks. The dual cluster head alternation mechanism is adopted to balance the energy consumption of cluster head nodes, and it helps to eliminate redundancy through the compressive sensing data fusion technology, and improve the network throughput of the sensor network effectively. The simulation results show that the proposed algorithm is able to enhance the networks performance, especially in reducing the number of lost packets, and prolonging the network's lifetime.
\end{abstract}

Keywords: Compressed sensing, Data fusion, Double cluster head alternation, Network load balance, Wireless Sensor Networks (WSNs).

\section{INTRODUCTION}

Wireless Sensor Network (WSN) is deployed in monitoring area that is composed of a large number of lowcost micro sensor nodes, which form multiple hops selforganizing network through wireless communication mode [1]. Sensor nodes in WSN will send perceived information to the base station through multiple hops communications. The base station transfers interactive information to the task management center through the mobile communication network, satellite communications network and Internet network, so as to realize the perception of the physical world and the control of the node. Sensor nodes are usually powered by batteries with limited energy, so batteries' energy should be saved as much as possible in the design of compressive sensing in each layer, so as to prolong the network life.

In a hierarchical sensor network, a variety of algorithms have been proposed for compressive routing sensing, such as: LEACH [2], HEED [3], the GAF algorithm [4], etc. As clustering routing maintenance overhead is small, the network topology has a good scalability, and with the increase of network scale, network has better performance in the form of clustering. LEACH was the first compressive sensing algorithm to put forward low energy consumption clustering routing compressive sensing. Network operation cycle is divided into several rounds, and in each round, network will be divided into multiple clusters, which can not only reduce the amount of communication data and balance energy consumption of network, but also prolong the network lifetime.

Literature [5] proposed a two-step cluster head election mechanism. First, elect temporary cluster number, which is more than the optimal value. Then, according to the residual energy of temporary cluster heads and the distance to the

*Address correspondence to this author at the School of Control and Computer Engineering, North China Electric Power University, 102206, China; Tel: +86-010-61772520; Fax: +86-010-61772568; E-mail: juyun1982@ncepu.edu.cn base station for further screening, make the number of network clusters close to the optimal. Although literature [5] obtained the optimal number of clusters, yet still can't solve the problem of uneven distribution of the number of nodes in the cluster (that is, the size of the cluster). Literature [6] proposed a mechanism of dual-cluster head. One cluster head is responsible for receiving a cluster node's perception data, besides it performs data aggregation. Then it sends this data in the direction of the base station. The other cluster head is responsible for the forwarding of data from low level cluster head to high level cluster head. Literature [6] proposed dual cluster head to reduce the burden of cluster head nodes, but both the cluster heads need to keep in the listening state in the process of work, and therefore the channel listener will also cost a lot of energy.

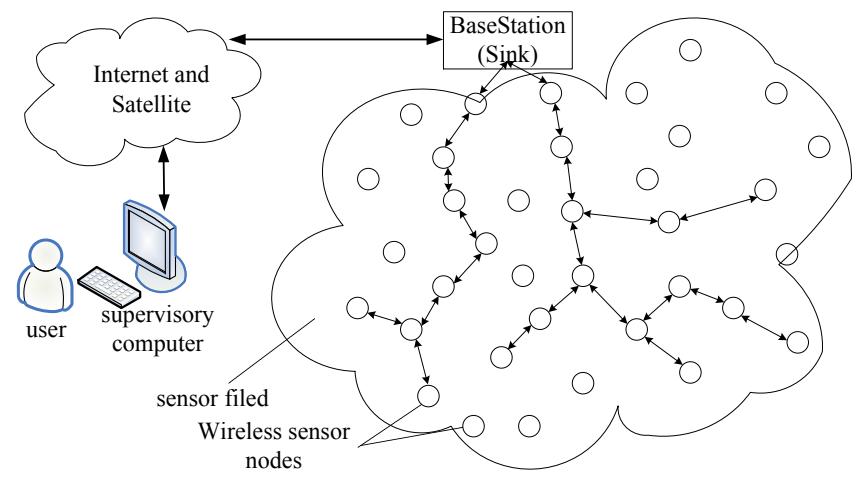

Fig. (1). Wireless sensor networks architecture.

According to the theory of compressed sensing (hereinafter referred to as CS) [7], as long as the signal is sparse, it can pass a few observations with high probability to reconstruct the original signal. Literature [8] pointed out that the Fourier coefficient of smooth signal, the wavelet coefficient, total variation norm of bounded variation function, Gabor coefficients of vibration signal and discrete Curvelet coefficients of the edge of the image signal all have 
enough sparse to reconstruct the original signal via compressive sensing theory. CS has excellent compression performance, the encode and decode codecs are independent of each other, and the encoding complexity is much lower than reconstructing signal decoding complexity. In WSN, the sensor node battery capabilities are very limited, only suitable for low complexity encoding, and that the base station with continuous energy supply and strong processing capacity, can perform more complex decoding operation. Therefore, CS is especially suitable for application in WSN.

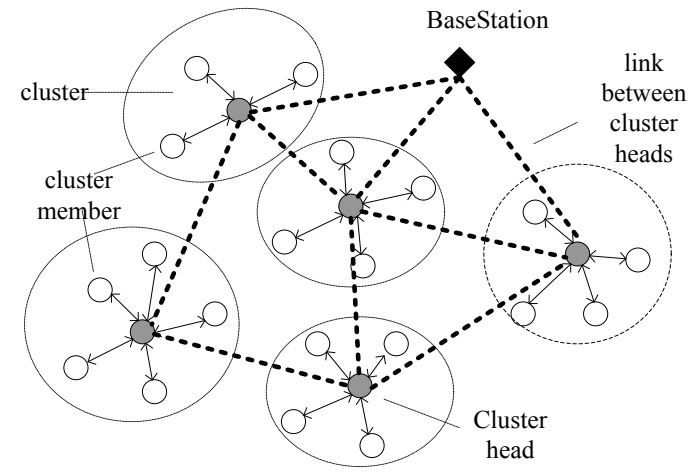

Fig. (2). Clustered network topology.

In this paper, combining with clustering topology of LEACH protocol, a WSN routing protocol based on a dual cluster head alternating and compressive sensing is proposed. Section 1 of this article introduced the compressive sensing theory. The second section describes the system model. Section 3 presents a detailed description of compressive sensing. Section 4 presents the simulation analysis.

\section{THE COMPRESSIVE SENSING THEORY}

This paper adopts the compressive sensing theoretical frameworks of literature [7-9], which have been widely used.

\subsection{Signal Sparse Representation}

Consider one dimensional real time signal $\mathrm{x}$, length $\mathrm{N}$, this $\mathrm{N}$ can be seen as an $\mathrm{R}^{\mathrm{N}}$ space $\mathrm{N} \times 1 \mathrm{~d}$ column, base vector for $\Psi_{\mathrm{i}}(\mathrm{i}=1,2, \ldots, \mathrm{N})$; the signal can be expressed as:

$x=\sum_{i=1}^{N} \alpha_{i} \Psi_{i}, x=\Psi \alpha$

where $x$ is the representation of signal in time domain, $\alpha$ is the representation of the signal in $\Psi$ domain. If there is only $\mathrm{K}$ nonzero value of $\alpha, \mathrm{K}<<\mathrm{N}$; if $\alpha$ according to the exponential decays after sorting and tends to be 0 , the signal can be thought of as sparse. Whether the signal is similar to sparse is the key to use compressive sensing theory.

\subsection{Measuring Coding}

Design a $M \times N(M<<N)$ dimensional matrix $\Phi$ to observe the signal, which is not related to transformation basis matrix, that get $\mathrm{M} \times 1$ dimensional measurement vector:

$\mathbf{y}=\boldsymbol{\Phi} \mathbf{x}=\boldsymbol{\Phi} \boldsymbol{\Psi} \alpha=\boldsymbol{\Theta} \alpha$

Among them, the choice of measurement matrix $\Phi$ has nothing to do with the signal $x$. Literature [8] pointed out that, currently the measurement matrices used for compressive perception are Gaussian random matrix, hard Fourier random matrix, Hadamard matrix, etc. After observation, the original signal reduced from $N$ to $M \mathrm{~d}$, the measured $K$ value retained the original signal information, so as to ensure the accuracy of signal reconstruction.

Because $M<<N$, that means the number of equation is less than the number of unknowns, so the equations in general will have no definite solution. However, because the sparse signal is $\mathrm{K}(K<<M)$, if $\Theta$ meets restricted isometric property (RIP), namely $\Phi$ measurement matrix and sparse matrix $\Psi$ are not related, then measured value $\mathrm{M}$ can be used to reconstruct the original signal.

\subsection{Signal Reconstruction}

If the signal $K$ is sparse, and $\Theta$ meets RIP criterion, then reconstruct signal by solving the minimum 11 norm:

$$
\min _{\alpha}\|\alpha\|_{11} \text { s.t. } y=\Theta \alpha=\Phi \Psi \alpha
$$

Literature [9] pointed out that when the number of Gaussian measurement $M \geq c \cdot K \cdot \log (N / K)$, which is subject to the independent identical distribution. $c$ is a small constant [9], it will restrict sparse vector $\mathrm{K}$ precisely by using the 11 norm. Then, this problem becomes a convex optimization problem, and can be solved by converting it to linear programming problem. The typical algorithms to reconstruct signals obtained by solving optimization problem are: BP (track), MP (Matching Pursuit), OMP (Orthogonal Matching Pursuit), StOMP orthogonal matching pursuit (section) and so on.

\section{SYSTEM MODEL}

\subsection{Network Model}

This paper assumes that the nodes in wireless sensor network within the target area are uniformly deployed in a square, and have the following features:

1. Stations deployed outside the area, with continuous energy supply, strong ability of processing power, wireless communication and storage capacity.

2. After the completion of the network deployment, perform a whole network position during initialization. And after initialization, the base station and all the nodes know their position, and each node saves the position information of neighbor nodes in the process of locating. The WSN positioning technology can be found in the literature [1];

3. Nodes manage their residual energy and wireless transmission power according to the regulations of the distance between the sender and the receiver.

4. MAC layer uses energy efficient MAC compressive sensing, regardless of the interference and collision caused by the retransmission.

\subsection{The Energy Consumption of Wireless Communication Model}

Energy consumption of wireless communication is a major part of energy consumption in WSN. This paper uses 
the energy consumption of wireless communication model that was presented in literature [10].

The document [10] shows that when the communication distance between nodes is less than the threshold $d 0$, by using the free space model, the sending data of consumption energy is proportional to the square of the distance; Otherwise using the multipath fading model, the sending data of consumption energy is directly proportional to the four square of the distance. The distance between the sender and the receiver is $d$, the transmitting energy consumption $k$ bit data can be expressed as:

$E_{\mathrm{Tx}}(k, d)=\left\{\begin{array}{c}k E_{\text {elec }}+\mathbf{k} \varepsilon_{\mathrm{fs}} \mathbf{d}^{2}, \mathbf{d}<\mathbf{d}_{\mathbf{0}} \\ \mathbf{k E} E_{\text {elec }}+\mathbf{k} \varepsilon_{\mathrm{mp}} \mathbf{d}^{\mathbf{4}}, \mathbf{d} \geq \mathbf{d}_{\mathbf{0}}\end{array}\right.$

where $E_{\text {elec }}$ is the energy for wireless transceiver circuit, $\varepsilon_{f s}$ and $\varepsilon_{m p}$ respectively represent the proportionality coefficient of energy consumption by power amplifier when $d<d_{0}$ and a $d \geq d_{0}$ or when the power amplifier, among them, $d_{0}$ can be expressed as:

$\mathbf{d}_{\mathbf{0}}=\sqrt{\frac{\varepsilon_{\mathrm{fs}}}{\varepsilon_{\mathrm{mp}}}}$

Energy consumption of the receiver to receive $\mathrm{k}$ bit data as:

$E_{\mathrm{Rx}}(\mathbf{k})=\mathbf{k} \boldsymbol{E}_{\text {elec }}$

\section{COMPRESSIVE SENSING DESCRIPTION}

The cluster head election mechanism proposed in LEACH with randomness, does not consider the location of nodes, and cannot guarantee cluster heads even distribution throughout the network. And there is large scale difference in different clusters. In order to improve the above problems, first, elect temporary cluster heads using distributed algorithm, using local information to optimize a neighbor cluster. Then using the centralized algorithm, according to residual energy and location information of member node, elect better cluster heads. In addition, compressive sensing theory is introduced to the WSN, which is used for data fusion of cluster nodes. Then the dual cluster head alternate mechanism has been put forward to further reduce the burden of cluster heads.

In compressive sensing, the network life cycle is divided into several rounds, and each round is divided into clusters and two phases of data transmission, as shown in Table $\mathbf{1 .}$

\subsection{Temporary Cluster Head Election}

Cluster head election mechanism of LEACH compressive sensing is as follows: At the beginning of each round, all the nodes are involved in the campaign of cluster. Set a threshold $T$, when electing the cluster head. Those which have not been elected for cluster head nodes in the round $1 / P$, will generate a random number Rand between
$0 \sim 1$. When Rand is less than the threshold $T$, the node will be elected as cluster head.

$T=\frac{\mathbf{P}}{1-\mathbf{P} \times\left(\boldsymbol{r} \bmod \frac{1}{\mathbf{P}}\right)}$

Among them, $P$ denotes the percentage of the network cluster head nodes of the total number of the node, $r$ denotes the current round number.

When electing temporary cluster head, use the distributed algorithm, which is similar to the LEACH. After the node elected to the temporary cluster head, it will issue a message, which contained the node's identity (referred to as "ID"), such as residual energy and location information. The nodes, which are not cluster head, will receive and store the cluster heads broadcast news, and will send a message to join with the nearest cluster according to the distance between it and each other cluster head.

\subsection{Neighbor Cluster Optimization}

In Section 3.1, the following problems occur during building temporary clusters: 1) the uneven distribution of cluster heads; 2) the size of each cluster has a large difference; 3) temporary cluster head election without considering the node's residual energy and location information. After the completion of building temporary cluster, neighbor cluster heads broadcast announcement messages to each other, which can obtain the number of nodes in each neighbor cluster. Temporary cluster heads use neighbor cluster optimization algorithm to optimize a neighboring clusters, so as to solve the problem of uneven clustering. The optimization goal is: the larger clusters compensate part of nodes for smaller clusters, so as to make cluster distribution more uniform, then the energy consumption of cluster heads is even more balanced.

Set $N_{0}$ as the number of member nodes in the local cluster, and $N_{i}$ as the number of member nodes in the neighbor cluster. Temporary cluster heads calculate the average $N_{\text {aver }}$ of nodes in all neighbor clusters, that $N_{\text {aver }}=1 /(n+1) \times\left(N_{0}+\sum_{i=1}^{n} N_{i}\right)$, where $n$ is the number of neighbor clusters. If $N_{0}>N_{\text {aver }}$, temporary cluster head starts the optimization algorithm. The algorithm is divided into the following steps:

1. Calculate the number of nodes available for compensation of $N_{c}^{0}=N_{0}-N_{\text {aver }}$;

2. Perform traversal operations, if $N_{i}<N_{\text {aver }}$, then add it to compensate set (referred to as CS), and calculate the number of nodes to be compensated, $N_{c}^{i}=N_{\text {aver }}-$ $N_{i}$

3. Count the total number of nodes to compensate for all neighbor clusters in the CS, $N_{c}=\sum_{j=1}^{n^{\prime}} N_{c}^{j}$.Among them, $n^{\prime}$ is the total number of neighbor clusters which need to be compensated for CS;

Table 1. Process of each round.

\begin{tabular}{|l|l|l|l|c|}
\hline \multicolumn{3}{|c|}{ Clusters Stage } & Data Transmission Phase \\
\hline \hline Temporary cluster head election & Neighbor cluster optimization & Cluster head election & TDMA announcement & The data transfer \\
\hline
\end{tabular}


4. Perform traversal operations, to calculate the number of nodes, $N_{a c}^{i}$, which actually gains compensation for each cluster $\mathrm{C}_{i}$ of CS, there is $N_{a c}^{i}=N_{c}^{0} \times \frac{N_{c}^{2}}{N_{c}}$;

5. Traverse all the neighbors in the CS clusters in turn. For each neighbor cluster $C_{i}$, find out the $N_{a c}^{i}$ nodes closest to the $C_{i}$, then compensate for $C_{i}$, and update ID of the compensation nodes of temporary cluster;

6. Broadcast announcement messages to all nodes which are compensated for the other neighbor clusters. The messages include compensation node ID, and the corresponding new temporary cluster ID. After receiving the message, node will leave the old cluster, and send a message to join new clusters.

After a neighbor cluster optimization, clusters in network which $N_{0}>N_{\text {aver }}$ will replace $N_{0}-N_{\text {aver }}$ nodes to compensate for other neighbor clusters that need to be compensated, according to the above algorithm, so number of nodes in each neighbor cluster is balanced roughly in the local scope.

\subsection{The Cluster Head Election and TDMA Time Slot Allocation}

\subsubsection{Cluster Head Election}

Since the temporary cluster head selected in section 3.1 is not the best, after the neighbor clusters optimization in section 3.2 , the size of local clusters is roughly the same as that of the neighbors. Subsequently, we can use a centralized algorithm to select better cluster heads, using the node residual energy and local information of the temporary cluster heads.

Define the energy threshold $E_{\text {elect, }}$ of the elected cluster head as the energy consumption in sending and receiving 50 packets, when it comes to electing cluster heads. If temporary cluster head's member node's energy is lower than $E_{\text {elect}}$, it can't join in the race. Define $\mathrm{C}$ as the competiveness of alternative node of the cluster, $\mathrm{E}$ as the remainder of the alternative node energy, $d_{\max }$ as the maximum distance from alternative node to the other nodes of the cluster, $d_{\text {aver }}$ as the average distance from alternative node to all the other nodes of the cluster, $d_{t o B S}$ as the distance from alternative node to the base station, $d_{0}$ as the distance threshold value of wireless communication energy consumption model, there are:

$$
C=\omega_{1} E+\omega_{2} \frac{d_{\text {max }}}{d_{\text {aver }}}+\omega_{3} \frac{d_{0}}{d_{t_{\text {toBS }}}}
$$

Among them, $d_{\text {aver }}=1 /(N-1) \times \sum_{i=1}^{N-1} d_{\text {to_node_ } i}$, $d_{\text {to_node } i} i$ is the distance from alternate node to the $i$ node in the cluster, $\mathrm{N}$ as the total number of nodes in cluster, $\omega 1, \omega 2$, $\omega 3$ as the weight coefficients, and $\omega 1+\omega 2+\omega 3=1$. When selecting the cluster head, the greater the residual energy of nodes, the smaller the average distance to all other nodes, the closer to the base station, the stronger the competitiveness becomes. Temporary cluster head node traverses all members, then selects node with maximum competitive $C$ as the cluster head.

Because of dual cluster heads alternate mechanism, that needs to select two kinds of cluster head (main cluster head and cluster heads), when the vice cluster is selected, nodes which have been elected as the main cluster head with energy lower than $E_{\text {elect }}$ node, are no longer in the race. The vice cluster head election competitiveness formula is the same as the main cluster heads'.

\subsubsection{TDMA Time Slot Allocation}

Main cluster head assigned time slot according to the number of cluster nodes $N$, cluster heads need extra time slot allocation for data fusion and routing between clusters. Set $N_{\text {frams }}$ as the number of frames processed by the node in each time slot, $N^{\prime}$ as additional time slot number of cluster heads, and there are $N^{\prime}=\left\lceil N / N_{\text {frams }}\right\rceil$, main and deputy cluster heads alternate sharing extra slot $N^{\prime}$, (dual cluster head alternate mechanism is introduced in the next section), the total slot number $=N+N^{\prime}$. After time slot allocation is completed, the temporary cluster heads create TDMA radio message. The message contains the main cluster ID, deputy cluster ID, cluster member nodes time slot allocation table, the total number of the slot, and according distance from the farthest node to adjust the quantity of the transmission power, and broadcast messages by using random retreat mechanism among cluster heads.

After receiving the TDMA message, member nodes set their own time slot according to the time slot allocation table, and set the time slot period according to the total number of slots. If their own ID is equal to the ID of the cluster head or the deputy head cluster, then they are selected as the cluster head nodes. In data transmission phase, nodes only send data when their own time slot wakes up, and turns to sleep to save energy in other time slot.

\subsection{The Data Transmission}

\subsubsection{Track Dual Cluster Head Alternately}

In order to reduce the burden of cluster head nodes, use the two cluster heads to share the task. If the two cluster heads keep listening state, it would lead to unnecessary energy consumption. Using dual cluster head alternate mechanism can not only reduce the burden of cluster head nodes, but also reduce unnecessary channel listener. Set time $\mathrm{T}$ as data transmission phase time cost in each round, $t_{\text {slot }}$ as each time slot, $T_{\text {slot }}$ as slot cycle, and $T_{\text {slot }}=\left(N+N^{\prime}\right) \times t_{\text {slot }}$, total number of time slot cycles in data transmission phase of each round is as follows:

$\mathbf{N}_{\text {slot }}=\frac{T}{T_{\text {slot }}}=\frac{T}{\left(N_{i j}+N_{i j}^{\prime}\right) \times t_{\text {slot }}}$

Dual cluster head alternate mechanism: set $\mathrm{m}$ time slot period into a group, $N_{\text {slot }}$ time slot period can be divided into $k$ groups, and $\mathrm{k}=\frac{N_{\text {slot }}}{m}$. When the $k$ is an odd number, the main cluster heads keep working, deputy for the common node cluster head degrade; When the $k$ is an even number, deputy cluster heads work normally, main cluster head degrades as the common nodes. So in the alternate cycle, nodes send sensory data to the main and assistant cluster head alternately. And since cluster head nodes are responsible for data fusion and routing between common clusters, so they effectively share the heavy task of cluster heads. $m$ is an awakening factor, if $m$ is 1 , the main and deputy cluster heads will alternate frequently. And frequently awakening the wireless communication module will cost extra energy; but if $\mathrm{m}$ is too big, when assistant cluster head is working, the main cluster heads need to sleep 
for a long time, that will affect the main cluster heads' dynamic management of clusters (e.g., replacement of the new cluster head, the addition of new nodes, etc.). Therefore, when dual cluster head alternate, the value of $m$ should be flexible according to specific application.

\subsubsection{Cluster Head Replacement Mechanism}

In the data transmission phase, if the cluster head nodes are dead, the packets of entire cluster will be lost, so it needs to use cluster head replacement mechanism when they need to change the new cluster head. The main cluster heads first check the residual energy in their own time slot, and if the energy is lower than the energy of the cluster head election threshold $E_{\text {elect }}$ (i.e. the energy consumption value when 50 packets are sent and received), then elect the main cluster again with the cluster head election algorithm described in the section 3.3.1. Node which has been selected as deputy cluster head drops out the race. After the election, main cluster head update messages should be broadcast, and then after being received by the members of cluster nodes, the cluster head will update.

By the same token, when the residual energy of deputy cluster heads is below $E_{\text {elect }}$, deputy cluster head sends replacement request to the main cluster head. After receipt of such a request, the main cluster heads will elect the deputy heads again, and then broadcast vice cluster heads updated message. After receiving this updated message, members of the cluster nodes update the cluster heads.

\subsubsection{Data Fusion}

Cluster heads use compression perception for data fusion. This paper adopts the research achievements of literature [9], measurement matrix by Gaussian random matrix, reconstruction algorithm by the OMP (Orthogonal Matching Pursuit). Set the packet length as $N_{\text {packet }}$, sparse degree as $K$, the number of observations as $M$. By literature [9], under the particular condition of Gaussian random measurement matrix and OMP reconstruction algorithm, when observation number $\mathrm{M}$ and sparse degree $\mathrm{K}$ meet the following condition, cluster head can be accurately reconstructed (ignoring reconstruction error in this article):

$$
\mathrm{M} \approx 2 \cdot \mathrm{K} \cdot \ln \left(\mathrm{N}_{\text {packet }}\right)
$$

In this article, take $N_{\text {packet }}=475, K=10^{[9]}, M \approx 123.27$, data fusion rate is $M / N \approx 26 \%$.

Compressed sensing has excellent compression performance. The importance of its application in this article is: 1) the coding complexity is low, easy implementation on sensor node; 2) the amount of data becomes less after data fusion, and route using the less energy; 3) at present most routing protocols assume that data fusion rate is fixed value, or assume that practicality is not strong, and thus compression perception theory has been introduced to data fusion. This paper makes the deal much more practical, which is of great significance.

\subsection{Routing Between Clusters}

When routing between clusters, cluster node $\mathrm{CH}_{0}$ needs to find the cluster head node with the minimum Routing Cost (referred to as RC) as the next jump. Routing process between clusters is divided into the following steps:

1. $\mathrm{CH}_{0}$ estimates the routing cost $R C_{0}$ for sending the packet directly to the base station;

2. Perform traversal operations, if the distance from neighbor cluster head to the base station is less than the distance from itself to the base station, add it to the routing table;

3. In the routing table, the candidate cluster heads $\mathrm{CH}_{i}$, estimate the energy consumption $T_{i}$ for $\mathrm{CH}_{0}$ to send the packet to $\mathrm{CH}_{i}$, the energy dissipations $\mathrm{R}_{i}$ for $\mathrm{CH}_{i}$ to receive packets, the energy dissipations $R C_{i}^{\prime}$ for $\mathrm{CH}_{i}$ to send the packet to the base station. Then the routing cost of $\mathrm{CH}_{0}$ for routing the packet to $\mathrm{CH}_{i}$, is $R C_{i}=T_{i}+R_{i}+R C_{i}^{\prime}$;

4. Traverse all candidate cluster heads in the routing table, record all $C H_{i}$ when $R C_{i}<R C_{0}$, the next-hop routing between clusters is one of the smallest $\mathrm{CH}_{i}$, if it has no $\mathrm{CH}_{i}$ found to meet the requirements, $\mathrm{CH}_{0}$ directly sends the packet to the base station.

\section{THE SIMULATION ANALYSIS}

We use OMNeT++ simulation software to simulate the protocol proposed in this paper, and use MATLAB to draw. In the simulation scenario, 100 sensor nodes are uniformly distributed within the square area of $100 \mathrm{~m}$ by $100 \mathrm{~m}$, the base station is located outside the area, with the coordinates $(50 \mathrm{~m}, 150 \mathrm{~m})$. The percentage of the number of cluster nodes in the network to the total number of nodes $P=5 \%$, the initial energy of nodes is $2 \mathrm{~J}$, and the packet length is 500 bytes, in which, 25 bytes are packet header, 475 bytes are valid data, data fusion rate $P_{d a}$ is $26 \%$. The energy consumption parameters of wireless communication in the model are taken from literature [10], that is $E_{\text {elec }}=50 \mathrm{~nJ} / \mathrm{bit}$, $\varepsilon_{f s}=10 \mathrm{pJ} / \mathrm{bit} / \mathrm{m}^{2}, \varepsilon_{m p}=0.0013 \mathrm{pJ} / \mathrm{bit} / \mathrm{m}^{4}, \mathrm{~d}_{0}=\sqrt{\varepsilon_{f s / \varepsilon_{m p}}} \approx 87.7 \mathrm{~m}$. Data fusion energy consumption $E_{D A}=5 \mathrm{~nJ} / \mathrm{bit}$. Set time for each round as $30 \mathrm{~s}$, clustering stage as $5 \mathrm{~s}$, the data transmission phase as $25 \mathrm{~s}$, each slot time as $0.03 \mathrm{~s}$. End condition of simulation is that all nodes were killed.

Fig. (4) makes a comparison between using the neighbor cluster optimization algorithm and not using the optimization algorithm. In the entire life cycle, the number of occurrences of cluster member nodes are counted (the size of a cluster) in the interval $[1,100]$.

As shown in Fig. (4), before optimization, cluster member nodes counts are mainly distributed in the interval $[1,40]$, with strong randomness, and the distribution of the size of the cluster is uneven. After optimization, 477 clusters in total are generated throughout the entire life cycle. When number of cluster member nodes get 20 they appear 373 times, and the size of the cluster distribution is rather more even. The improvement of the cluster performance is obvious.

We test and verify the performance of dual cluster head alternate mechanism, to use or not to use the mechanism for simulation respectively. Fig. (5) shows the two kinds of simulation mechanisms certain with specific percentage of dead nodes. Fig. (5) shows that, in dual cluster head alternate 


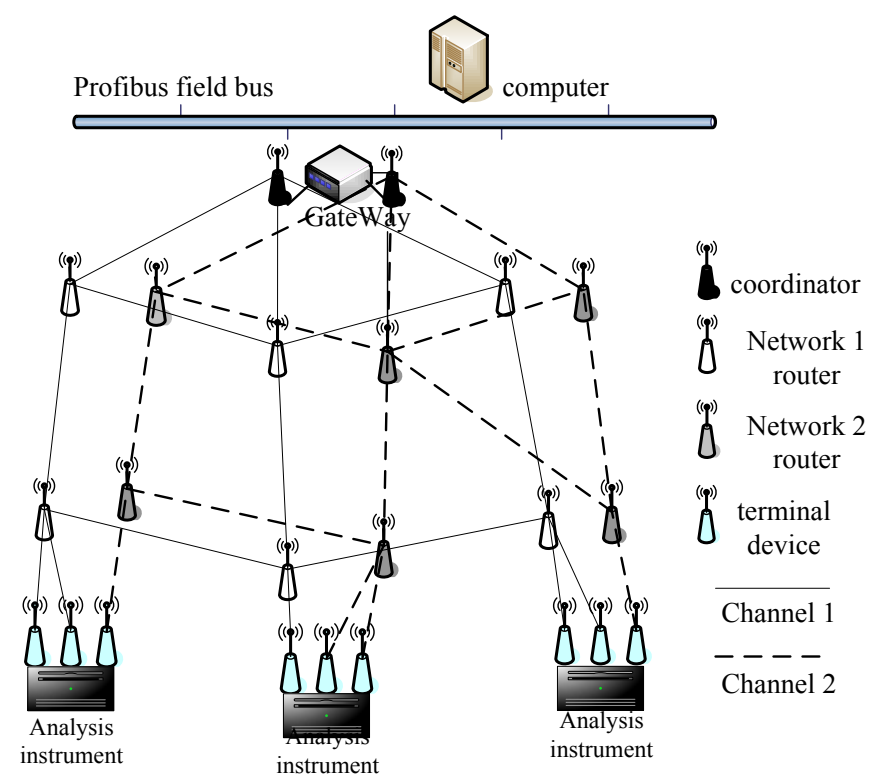

Fig. (3). Double-network redundant hot-standby schematic.

mechanism, first node death round number is the $82^{\text {th }}$ round, half nodes death round number is the $102^{\text {th }}$ round, all the nodes death round number turns to the $109^{\text {th }}$; above parameters for the single cluster head mechanism are the $76^{\text {th }}, 97^{\text {th }}, 102^{\text {th }}$ round respectively. Compared with the single cluster head mechanism, dual cluster head alternate mechanism prolongs the network life. The reasons for this improvement include: because data fusion and routing between clusters will spend a lot of energy, dual cluster head alternate mechanism adopts two cluster heads working alternately, effectively splitting the heavy task of the cluster heads.

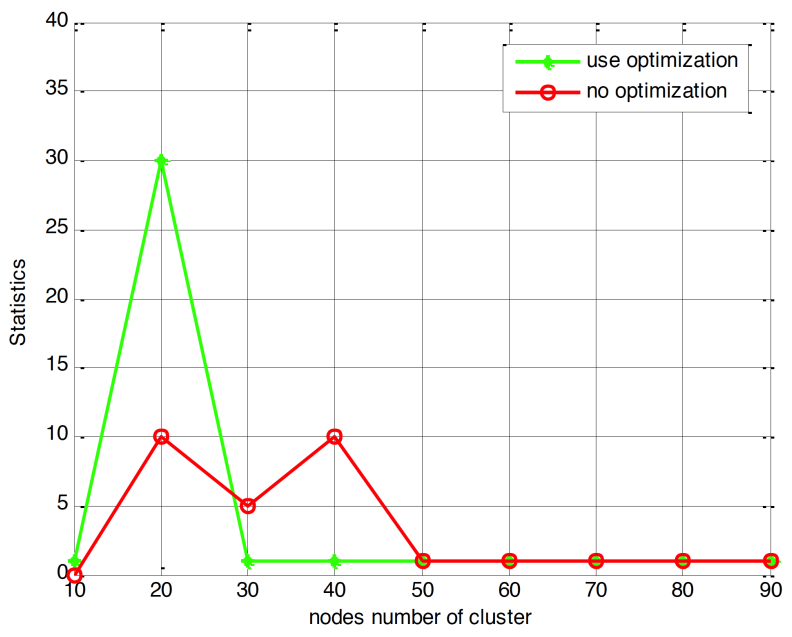

Fig. (4). Statistics of cluster's size.

To verify the performance of the cluster head replacement mechanism, Fig. (6) shows the number of lost packets due to death of cluster nodes under the condition of using the cluster head replacement mechanism and not using the replacement mechanism.

Fig. (6) shows that without the cluster head replacement mechanism, the total number of lost packets due to the death of cluster heads reaches to 7788 packets. Adding the cluster head replacement mechanism, the number of lost packets sharply reduces, indeed drops to 324 .

In addition, this paper adds the simulation to LEACH protocol and the improved GAF algorithm, and then compared with DHLCS.

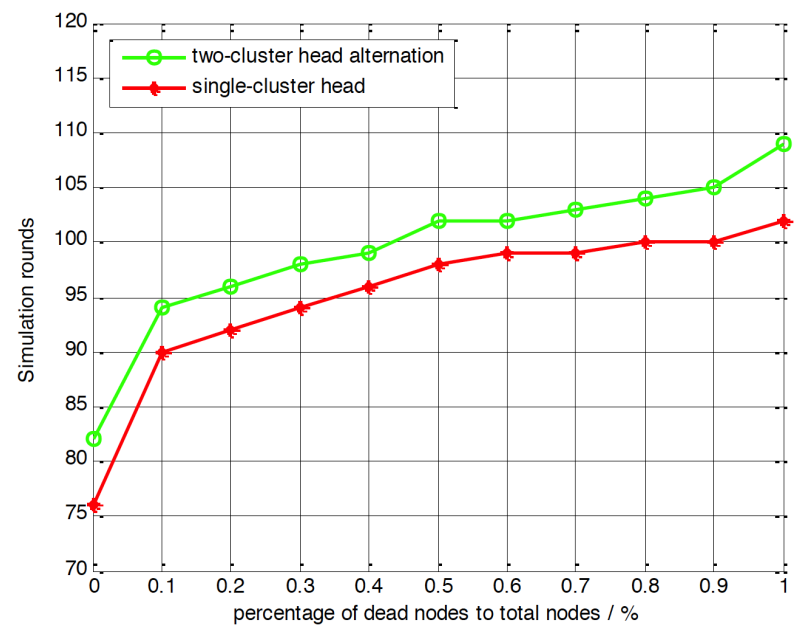

Fig. (5). Comparison of simulation rounds between two-cluster head and single-cluster head when specific percentage of nodes are dead.

Fig. (7) calculates the round number of the three kinds of protocols when specific percentage nodes are dead. Fig. (7), compared LEACH protocol and improved algorithm of GAF. DHLCS can delay the death of the first node number, and has a better performance when the percentage of dead nodes is less than $50 \%$, and can balance the network energy consumption (curve smoother). After 50 to $70 \%$ of the nodes die, although improved GAF algorithm and LEACH still have better performance, yet because of the limited communication distance of each node, the network will be formed as the subnet cut off from the base station. And the data of the remaining survival nodes will not be able to reach the station, that will cause the waste of network resources. The 
reasons for why DHLCS can improve the performance are as follows: the neighbor cluster optimization algorithm makes the size of the cluster more balanced, dual cluster head alternate mechanism reduces the burden of cluster heads, energy efficient routing algorithm between the election of cluster head and cluster further reduces the network energy consumption. Therefore, DHLCS can balance the network energy consumption and prolong network lifetime.

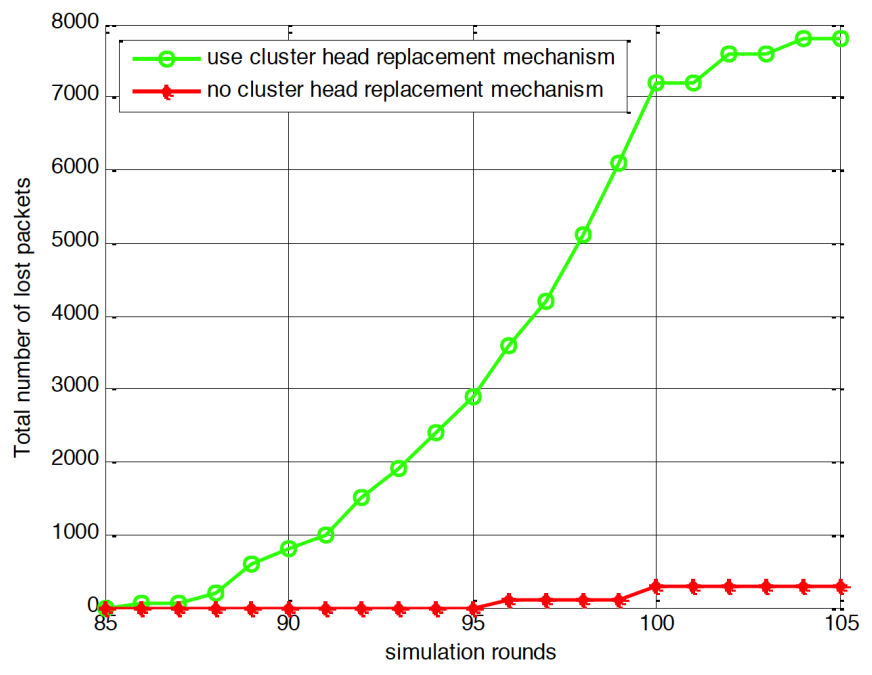

Fig. (6). Total number of lost packets due to the death of cluster head.

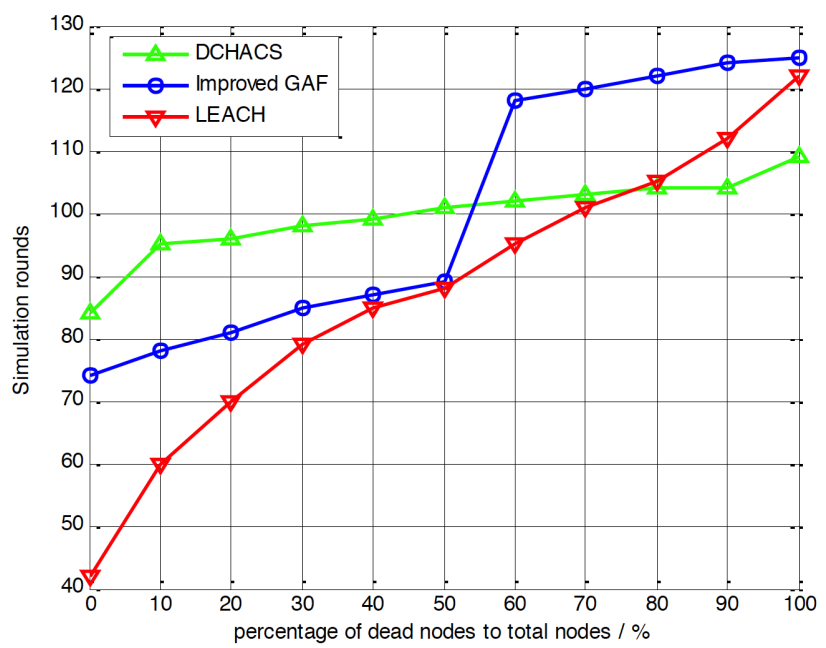

Fig. (7). Comparison of simulation rounds of three protocols when specific percentage of nodes are dead.

\section{CONCLUSION}

This paper proposes a WSN routing protocol (DHLCS) based on dual cluster head alternate mechanism and compressive perception. DHLCS two-step cluster head election mechanism is used to elect the optimal cluster head. The neighbor cluster optimization algorithm dynamically adjusts the cluster size by using local information; dual cluster head alternate mechanism is used to share the burden of cluster heads. Besides, DHLCS adopts cluster head replacement mechanism to make the new cluster head to replace the dead cluster head. The simulation shows that DHLCS can obtain a better performance of clusters, make each cluster size distribution more uniformly, and sharply reduce the number of lost packets due to the death of cluster heads, balancing the network energy consumption. But in terms of the number of clusters of each round, the protocol proposed by literature [6] is better than that of DHLCS. And furthermore, another DHLCS neighbor cluster optimization algorithm has been proposed. While it is possible to make each cluster size distribution more uniformly, it does not take account of hotspots near the base station when using multi-hop transmission forward. Further work promises to study non-uniform optimization algorithm, to make the cluster near the base station having more nodes, so as to further improve the performance of the network.

\section{ACKNOWLEDGEMENTS}

This work is supported by the Fundamental Research Funds for the Central Universities of China (2016MS35).

\section{REFERENCES:}

[1] L.M. Sun, J.Z. Li, Y. Chen, and H.S. Zhu, Wireless sensor networks, Tsinghua University Press, Beijing, 2005.

[2] W. R. Heinzelman, A. Chandrakasan, and H. Balakrishnan, "Energyefficient communication protocol for wireless microsensor networks," In: Proceedings of the $33^{\text {rd }}$ Annual Hawaii International Conference on System Sciences, vol. 2, p. 10, 2000.

[3] O. Younis and S. Fahmy, "HEED: a hybrid, energy-efficient, distributed clustering approach for ad hoc sensor networks," In: IEEE Transactions on Mobile Computing, vol. 3, pp. 366-379, 2004.

[4] P. Santi and J. Simon, "Silence is golden with high probability: Maintaining a connected backbone in wireless sensor networks," In: Wireless Sensor Networks, Springer, Berlin, 2004, pp. 106-121.

[5] Z.G Sun, Z.W. Zheng, and S.J. Xu, "An efficient routing protocol based on two step cluster head selection for wireless sensor networks". In: Proceedings of the $5^{\text {th }}$ International Conference on Wireless Communications, Networking and Mobile Computing, IEEE Press, Beijing, pp. 1-5, 2009.

[6] S. Ebadi, M. Ghasembaglou, A. H. Navin, and M. K. Mirnia, "Energy balancing in wireless sensor networks with selecting two cluster-heads in hierarchical clustering," In: International Conference on Computational Intelligence and Communication Networks (CICN), 2010, pp. 230-233.

[7] D. L. Donoho, "Compressed sensing", In: IEEE Transactions on Information Theory, vol. 52, pp. 1289-1306, 2006.

[8] G.-M. Shi, D.-H. Liu, D.-h. Gao, Z. Liu, J. Lin, and L.-J. Wang, "Advances in theory and application of compressed sensing," Acta Electronica Sinica, vol. 37, pp. 1070-1081, 2009.

[9] L. Xiao-bo, Research on measurement matrix based on compressed sensing, Beijing Jiaotong University, Beijing, 2010.

[10] C.L. Zhao, S. Mao, H. Tan, "An energy-balanced clustering protocol for wireless sensor network", Radio Engineering of China, vol. 41, no. 3,2011 . 